\title{
ANÁLISE DA GESTÃo AMBIENTAL NOS INSTITUTOS FEDERAIS DE EDUCAÇÃO, CIÊNCIA E TECNOLOGIA
}

\author{
Aurélio Ferreira Borges ${ }^{1}$, José Luiz Pereira de Rezende², Luís Antônio Coimbra Borges ${ }^{3}$, \\ Rosângela Alves Tristão Borém ${ }^{4}$, Renato Luiz Grisi Macedo ${ }^{5}$, Maria dos Anjos Cunha Silva Borges ${ }^{6}$
}

(recebido: 4 de abril de 2011; aceito: 25 de janeiro de 2013)

RESUMO: Conduziu-se este trabalho, com o objetivo central de analisar o perfil ambiental dos Institutos Federais de Educação, Ciência e Tecnologia (IF), quando analisadas as práticas de gestão ambiental. Para atingir esse objetivo foi desenvolvida uma entrevista por intermédio de questionário, o qual foi respondido por 82 Campi do Brasil, de um total de 230, de forma a poder diagnosticar práticas de gestão ambiental nesse setor. Com base na avaliação do grau de implantação de determinadas vertentes e práticas de gestão ambiental, foi traçado o perfil ambiental dos IF. As práticas de gestão ambiental são meios pelos quais as organizações podem melhorar o seu desempenho. Um comportamento ecoeficiente otimiza o uso dos recursos e evita a produção de resíduos, permitindo poupanças significativas. Um bom desempenho ambiental evita custos resultantes da aplicação do princípio do poluidor-pagador. Este trabalho constituiu uma base de apoio aos diversos Campi dos IF, sugerindo quais as vantagens de recorrer a práticas de gestão ambiental, de forma a aumentar o desempenho ambiental nessas instituições de ensino, pesquisa e extensão públicas. Concluiu-se que não há gestão ambiental nos Institutos Federais de Educação, Ciência e Tecnologia que preencha os requisitos da sustentabilidade e que o Índice de Avaliação Ambiental dos Institutos Federais de Educação, Ciência e Tecnologia (IADAIFE) para quatro Campi foi muito fraco e para 78 Campi foi fraco.

Palavras-chave: Gestão ambiental, desempenho ambiental, Institutos Federais de Educação, Ciência e Tecnologia.

\section{ANALISIS OF ENVIRONMENTAL MANAGEMENT IN FEDERAL INSTITUTE OF EDUCATION, SCIENCE AND TECHNOLOGY}

ABSTRACT: The main objective was to analysis the environmental profile of the Federal Institutes of Education, Science and Technology (IF), when analyzing the environmental management practices. To achieve this goal was developed through an interview questionnaire which was answered by 230 Campuses in Brazil, in order to diagnose environmental management practices in this sector. Based on the assessment of the degree of implantation of certain aspects and environmental management practices was traced the environmental profile of the IF. The environmental management practices are the means by which organizations can improve their performance. An eco-efficient use of resources prevents waste generation and enables significant savings. An adequate environmental performance avoids costs resulting from the application of the polluter-pays principle. This work provided a baseline to support several of IF Campus, suggesting the advantages of using environmental management practices in order to improve environmental performance in these public educational, research and extension institutions. It was concluded that there is no environmental management in the Federal Institutes of Education, Science and Technology that meets the requirements of sustainability and that the Environmental Evaluation Index of the Federal Institutes of Education, Science and Technology (IADAIFE) for four Campi was very weak and for 78 Campi was weak.

Key words: Environmental management, environmental performance, the Federal Institutes of Education, Science and Technology.

\footnotetext{
${ }^{1}$ Licenciado em Ciências Agrícolas, Professor Pós-Doutorando em Engenharia Florestal - Instituto Federal de Educação Ciência e Tecnologia de Rondônia/IFRO - Rodovia RO 399, Km 05 - 76.993-000 - Colorado do Oeste, RO, Brasil - aferreiraborges@yahoo.com.br

${ }^{2}$ Engenheiro Florestal, Pós-Doutor em Ciências Florestais - Universidade Federal de Lavras/UFLA - Departamento de Ciências Florestais - Cx. P. 3037 -37.200-000 - Lavras, MG, Brasil - jlprezen@dcf.ufla.br

${ }^{3}$ Engenheiro Florestal, Professor Doutor em Ciências Florestais - Universidade Federal de Lavras/UFLA - Departamento de Ciências Florestais Cx. P. 3037 -37.200-000 - Lavras, MG, Brasil - luis.borges@dcf.ufla.br

${ }^{4}$ Engenheira Florestal, Professora Doutora em Produção Vegetal - Universidade Federal de Lavras/UFLA - Departamento de Biologia - Cx. P. 3037 -37.200-000 - Lavras, MG, Brasil - tristao@dbi.ufla.br

${ }^{5}$ Engenheiro Agrônomo, Pós-Doutor em Engenharia Florestal - Universidade Federal de Lavras/UFLA - Departamento de Ciências Florestais - Cx. P. 3037 - 37.200-000 - Lavras, MG, Brasil - rlgrisi@dcf.ufla.br

${ }^{6}$ Licenciada em Letras, Professora Mestre em Letras - Boticário Franchising S/A. - Rua Potiguara, 3744, Centro - 76993-000 - Colorado do Oeste, RO, Brasil-maria.cunhaborges@gmail.com
}

Cerne, Lavras, v. 19, n. 2, p. 177-184, abr./jun. 2013 


\section{INTRODUÇÃO}

O desempenho ambiental é expressão frequentemente utilizada em contextos diferentes, com objetivos distintos e diferentes significados. Por conseguinte, pode refletir diferentes problemas, tais como: a tendência para melhorar o meio ambiente, o estado do meio ambiente, a eficiência do meio ambiente, o cumprimento das normas ambientais, entre outros. Apesar dos diferentes significados, uma série de ferramentas de gestão ambiental utilizam a expressão desempenho ambiental: auditoria ambiental, avaliação de impacto ambiental, avaliação de risco ambiental, sistemas de gestão ambiental (SGA), entre outros (RAMOS et al., 2009).

Conforme esses autores, com a publicação da norma internacional ISO 14.031:1999, pela Organização Internacional de Normalização (ISO), o conceito de Avaliação de Desempenho Ambiental (ADA) foi aceito como uma ferramenta de gestão autônoma do meio ambiente. Essa norma integra as 14.000 famílias de normas ambientais internacionais e não é utilizada para fins de certificação ou de registro. Segundo a norma ISO 14.031:1999 (ASSOCIAÇÃO BRASILEIRA DE NORMAS TÉCNICAS - ABNT, 1999), a ADA é um processo que pretende facilitar a decisão dos gestores em relação ao desempenho ambiental de uma organização, selecionando indicadores ambientais, analisando dados, avaliando informações originárias do relatório de desempenho ambiental, utilizando de critérios técnicos e revisando periodicamente esse processo.

Conforme essa norma, a ADA é apresentada como um processo interno de gestão, uma ferramenta organizada para fornecer aos gestores instrução confiável e de fácil averiguação com fundamentação contínua, de forma a precisar em que ocasião o desempenho ambiental de uma organização está a desempenhar os critérios estabelecidos pela gestão. No entanto, essa definição é uma tarefa difícil. Por exemplo, a definição da norma ISO 14.031:1999 difere do que consta na norma ISO 14.001:2004.

Neste trabalho uma definição mais ampla de ADA é pretendida. O desempenho ambiental é aqui entendido como parte do desempenho de gestão e avaliação global. A definição ampla de indicadores de desempenho ambiental é igualmente assumida. AADA pode ser aplicada a todos os tipos de organizações e setores (privado ou público), independentemente do tipo, da dimensão, da complexidade, do país ou da região. As principais organizações privadas estão começando a avaliar e relatar os seus desempenhos ambientais e sociais, assim como fazem com o seu desempenho financeiro. Eficiência e competitividade, marketing, imagem pública, requisitos legais e compromissos internacionais são fatores que podem explicar esta tendência. No setor público, embora a evolução seja mais lenta, a ADA também está se tornando uma realidade crescente.

A adoção de práticas de ADA por agências regulatoras de Portugal, tanto no setor privado quanto no público, só são realizadas voluntariamente, sem qualquer regulamentação obrigatória. Algumas empresas portuguesas já publicam seus relatórios de desempenho ambiental e sustentabilidade, tanto em relatórios impressos como versões na Internet. Portugal adotou a Recomendação da Comissão Europeia, de 30 de Maio de 2001 (2001/453/ $\mathrm{CE})$ que trata do reconhecimento, mensuração e divulgação de questões ambientais nas contas anuais e relatórios anuais de empresas. Essa recomendação propõe a publicação de regulamento sobre as contas nacionais ambientais pelas autoridades portuguesas que tratam das normas de contabilidade (RAMOS et al., 2009).

$\mathrm{O}$ conceito de gestão ambiental pública ressalta o aspecto conciliador do Estado quanto às questões ambientais. Assim declara Floriano (2007, p. 2):

Gestão ambiental pública é um processo de mediação de interesses e conflitos entre atores sociais que agem sobre os meios físico-natural e construído. Este processo de mediação define e redefine, continuamente, o modo como os diferentes atores sociais, através de suas práticas, alteram a qualidade do meio ambiente $\mathrm{e}$ também, como se distribuem na sociedade os custos e os benefícios decorrentes da ação destes agentes.

Com as diretrizes da Agenda 21, da Declaração do Rio de 1992 e com o conceito de sustentabilidade elaborado por Carlowitz, em 1713, as políticas públicas de gestão ambiental devem ter como objetivo não só a gestão de recursos para proteger o ambiente natural, mas, principalmente, servir como orientação na solução de conflitos sociais que envolvam questões ambientais, tendo em vista o bem-estar social e a conservação de recursos para as futuras gerações (GROBER, 2010).

Gestão Ambiental (GA) é a supervisão da prática de atividades econômicas e sociais de forma a empregar de maneira racional os recursos naturais, renováveis ou

Cerne, Lavras, v. 19, n. 2, p. 177-184, abr./jun. 2013 
não. Conforme Nogueiro (2008), a gestão ambiental deve ter em vista o uso de práticas que cubram a permanência e preservação da biodiversidade, a reciclagem das matériasprimas, a diminuição do impacto ambiental das atividades humanas e o cumprimento da legislação ambiental sobre os recursos naturais. Segundo o autor, completa também a estrutura de conhecimentos pertinentes à gestão ambiental as técnicas para a recuperação de áreas degradadas, técnicas de reflorestamento, procedimentos para a exploração sustentável de recursos naturais, e o esboço de riscos e impactos ambientais para a estimação de novos empreendimentos ou ampliação de atividades produtivas e educacionais.

A gestão ambiental vem ganhando espaço crescente no meio empresarial. $\mathrm{O}$ desenvolvimento da consciência ecológica em diferentes camadas e setores da sociedade mundial acaba por envolver também o setor da educação. Ela classifica as atividades humanas para que estas acarretem o mínimo de impacto sobre o meio ambiente, desde a preferência das melhores técnicas até o implemento da legislação ambiental e a alocação correta de recursos humanos e financeiros (DRUZZIAN; SANTOS, 2006). Desse modo, o consumo irrefreável dos recursos naturais e a degradação do meio ambiente passaram a estabelecer atuações corretivas de ampla capacidade.

Segundo teoria proposta por Gomes (2006), a abordagem da questão ambiental em formato setorial e multidisciplinar, estudos de caráter técnico em detrimento dos aspectos epistemológicos e metodológicos dificultam a incorporação das Universidades brasileiras à dimensão ambiental e à formação de recursos humanos.

As Instituições de Ensino Superior introduziram a temática ambiental em seus diagramas de gestão a partir dos anos 1960. As primeiras experiências surgiram nos Estados Unidos, simultaneamente com as promoções de profissionais nas ciências ambientais, que se estenderam ao longo dos anos 1970.

Em agosto de 1993, na conclusão da conferência da associação das universidades comunitárias na Suécia, participantes de 400 universidades, de 47 países diferentes, focalizaram o tópico dos povos e do meio ambiente. A questão era encontrar maneiras de as universidades comunitárias, de seus líderes e de os estudantes acoplarem aos seus projetos metodologias para responder ao desafio da sustentabilidade. A reunião na Suécia, inspirada pelos exemplos de Talloires e de Halifax, e decepcionada pela fraca presença das universidades na Agenda 21, adicionou sua voz àquela mundial, que é concernida sobre a degradação do meio ambiente e do aumento da pobreza (THE SWANSEA..., 1993).

De acordo com Barata et al. (2007), existe a necessidade de se implantar um sistema de gestão ambiental nos órgãos da administração pública. Esses autores expõem alguns argumentos de caráter econômico, referentes aos benefícios obtidos por iniciativas privadas que adotaram critérios de ecoeficiência que, em si, seriam suficientes para justificar a implantação de uma política efetiva de gestão ambiental nas instituições públicas. Destaca-se a relevância da criação da Agenda Ambiental na Administração Pública, que pretendeu instaurar uma nova cultura institucional, visando à mobilização dos servidores para a otimização dos recursos, para o combate ao desperdício e para a busca de uma melhor qualidade do ambiente de trabalho.

A utilização de indicadores ambientais apresentase, atualmente, como uma ferramenta essencial na gestão e avaliação ambiental. Admite-se que os passos futuros dos indicadores ambientais passarão pela tentativa de atingir o mesmo estádio de maturidade de alguns indicadores econômicos e sociais. Mais importante do que o tipo de instrumento de gestão ambiental implementado, o que é relevante é focar o impacto ambiental das atividades e perceber se houve ou não melhoria de desempenho (RAMOS, 2004).

Segundo Tauchen e Brandli (2006), existem razões significativas para implantar um SGA numa Instituição de Ensino Superior, entre elas o fato de que os Institutos Federais de Educação e universidades podem ser comparadas com pequenos núcleos urbanos, envolvendo diversas atividades de ensino, pesquisa, extensão e atividades referentes à sua operação por meio de bares, restaurantes, alojamentos, centros de conveniência, entre outras. Para esses autores, um campus precisa de infraestrutura básica, redes de abastecimento de água e energia, redes de saneamento e coleta de águas pluviais e vias de acesso.

Bonnet (2002) realizou pesquisa numa universidade localizada na região de Bordeaux, na França, para identificar os consumos de energia e água disponíveis na instituição. Com relação ao consumo per capita de água, foi constatado que é o mais elevado, se comparado ao consumo médio das grandes cidades. Conforme o autor, o fato se agrava em virtude de parte da água consumida no Campus ser proveniente de aquíferos. Quanto ao consumo de energia, os parâmetros permaneceram semelhantes ao consumo dos habitantes das cidades, o que evidencia a necessidade de controle também desse item.

Cerne, Lavras, v. 19, n. 2, p. 177-184, abr./jun. 2013 
O objetivo deste trabalho foi analisar o desempenho ambiental dos IF brasileiros.

\section{MATERIAL E MÉTODOS}

Como instrumento de observação indireta, foi realizada pesquisa com emprego de questionário com 38 perguntas.

A administração dos questionários foi concretizada por meio do envio para os diretores-gerais de 230 Campi dos IF. Para situações de esclarecimento de dúvidas, foram disponibilizados os contactos do autor da proposta de pesquisa.

Foram enviados inicialmente 230 questionários a 230 Campi, por correio eletrônico. Por esse método de envio, foram recebidos três questionários de apenas três Campi. Posteriormente, adotou-se o método de envio via correio postal. Por esse método, foram enviados 227 questionários a 227 Campi, impressos em papel. Foram recebidos por esse método, 79 questionários de 79 Campi. Com os três questionários recebidos por e-mail mais os 79 recebidos via correio postal, o total de questionários recebidos foi 82 (Tabela 1 ).

Tabela 1 - Número de questionários enviados aos Campi e total de questionários recebidos, Brasil, em 2010.

Table 1 - Number of questionnaires sent to the Campuses and total number of questionnaires received, Brazil in 2010.

\begin{tabular}{|c|c|c|c|c|}
\hline $\begin{array}{c}\text { Questionários } \\
\text { enviados }\end{array}$ & $\begin{array}{l}\text { № de } \\
\text { Campi }\end{array}$ & $\begin{array}{l}\text { Método de } \\
\text { envio }\end{array}$ & Recebidos & Total \\
\hline 230 & 230 & E-mail & 03 & 03 \\
\hline 227 & 227 & $\begin{array}{c}\text { Correio } \\
\text { postal }\end{array}$ & 79 & 79 \\
\hline $\begin{array}{l}\text { Total geral } \\
\text { recebido }\end{array}$ & & & & 82 \\
\hline
\end{tabular}

Fonte: dados da pesquisa.

A versão definitiva do questionário ocorreu após recomendações dos membros da banca de qualificação de Doutorado, nomeadamente no que diz respeito à compreensibilidade, clareza e aceitabilidade.

Exemplos de perguntas do questionário:

a) Como classifica o desempenho ambiental do Campus do IF?

b) Existe no Campus uma estrutura responsável exclusivamente pelas questões ambientais?

c) Em caso afirmativo à questão anterior, qual a sua denominação?

Cerne, Lavras, v. 19, n. 2, p. 177-184, abr./jun. 2013

\subsection{Questionário práticas de gestão ambiental nos IF}

O questionário sobre práticas de gestão ambiental nos IF foi elaborado de forma a permitir diagnosticar as práticas de gestão ambiental no setor alvo, permitindo conduzir ao diagnóstico de um perfil para a GA.

O questionário foi composto por 38 questões fechadas subdivididas em subitens e agrupadas em categorias. As questões foram elaboradas a partir de teoria sugerida por Nogueiro (2008). A população estatística pesquisada totalizou a escala nacional da maior parte dos IF, ou seja, 37 Instituições representadas por 230 Campi, cuja amostra foi de 82 Campi, que representa taxa de resposta de 35,65\% (Tabela 2). Conforme Nogueiro (2008), considera-se $31 \%$ uma taxa de resposta adequada para esse tipo de pesquisa.

Tabela 2 - Taxa de resposta dos Campi dos IF, Brasil, em 2010.

Table 2 -Response rate of the Campuses of IF, Brazil in 2010.

\begin{tabular}{cccc}
\hline № de IF & População & Amostra & Resposta (\%) \\
\hline 37 & 230 & 82 & 35,65 \\
\hline
\end{tabular}

Fonte: dados da pesquisa usando o software R(R DEVELOPMENT CORE TEAM, 2008).

\subsection{Metodologia estatística}

A verificação da adequação da amostra à análise fatorial foi avaliada pelo teste de esfericidade de Bartlett (1954). O teste indica se as correlações entre as variáveis foram significativas ou não. Quando significativo, indica que as variáveis dependentes quantitativas presentes no Questionário que Diagnostica Práticas de Gestão Ambiental nos IF estão intercorrelacionadas e, portanto, existe sentido desenvolver uma análise fatorial.

A estatística descritiva foi utilizada para analisar os resultados. Chi-quadrado foi calculado para testar associações entre a frequência de distribuições entre os IF.

A fim de combinar os resultados obtidos em cada questão do questionário de ADA, um índice, o IADAIFE, foi desenvolvido para processar as informações de forma simplificada e útil, tomando como base teoria proposta por Ramos et al. (2009) e Ramos e Melo (2006), que desenvolveram o índice de avaliação do Desempenho Ambiental das Forças Armadas (DAFA) e Nogueiro (2008), que desenvolveu o Índice de Avaliação Ambiental dos Municípios de Portugal (ADAM). O IADAIFE dá uma visão global do conhecimento, da conscientização e da prática da ADA nos Campi dos IF brasileiros. 
Para o cálculo do IADAIFE, foi realizado cálculo com base na seleção de nove variáveis sobre práticas ambientais adotadas nos Campi dos IF (Tabela 3).

Tabela 3 - Variáveis utilizadas para o cálculo do índice IADAIFE, Brasil, em 2010.

Table 3 - Variables used to calculate the IADAIFE index, Brazil, in 2010 .

\begin{tabular}{ll} 
X1 & $\begin{array}{l}\text { Existência de estrutura responsável exclusivamente } \\
\text { pelas questões ambientais. }\end{array}$ \\
X2 & $\begin{array}{l}\text { Existência de profissional da área ambiental } \\
\text { responsável pela GA das instalações do Campus. }\end{array}$ \\
X3 & $\begin{array}{l}\text { Existência de ações de formação para os servidores, } \\
\text { sobre GA na Instituição. }\end{array}$ \\
X4 & $\begin{array}{l}\text { Implantação de Sistema de Gestão Ambiental. } \\
\text { Xlaboração de Relatórios Ambientais/ }\end{array}$ \\
X6 & $\begin{array}{l}\text { Utilização de Indicadores de Desempenho } \\
\text { Ambiental. }\end{array}$ \\
X7 & $\begin{array}{l}\text { Realização de Auditoria/Diagnóstico Ambiental. } \\
\text { Xtilização de Critérios Ambientais nas Compras }\end{array}$ \\
X8 & $\begin{array}{l}\text { Implicas. } \\
\text { de Desenvolvão da Agenda 21 Local ou outra estratégia }\end{array}$ \\
\hline
\end{tabular}

Fonte: adaptado de Nogueiro (2008), Ramos et al. (2009) e Ramos e Melo (2006); utilizando o software R (R DEVELOPMENT CORE TEAM, 2007).

Essa escala para o IADAIFE variou entre zero (o pior perfil ambiental) e um (o melhor perfil ambiental). Esse índice foi calculado através da seguinte equação:

IADAIFE $=\sum_{\mathrm{j}=1}^{\mathrm{m}} \frac{\sum_{\mathrm{i}=1}^{\mathrm{n}} \mathrm{Pi}}{\mathrm{m}}$

Onde,

$\mathrm{Pi}=$ Pontuação atribuída à variável $i$;

$\mathrm{n}=$ número total de $i$ variáveis, $\mathrm{i}=\mathrm{um}, \ldots$, nove

$\mathrm{m}=$ número total de $j$ Campi, $\mathrm{j}=\mathrm{um}, \ldots, 230$

Foram estabelecidas cinco categorias para classificar o desempenho ambiental fornecido pelo IADAIFE, numa escala de zero a um (muito fraco: $0-0,20$; fraco: 0,21 - 0,40; médio: 0,41-0,60; bom: $0,61-0,80$; muito bom: $0,81-1)$.

O software utilizado para as análises foi o R.2.10.1 (R DEVELOPMENT CORE TEAM, 2007).

\section{RESULTADOS E DISCUSSÃO}

A verificação da adequação da amostra à análise fatorial foi avaliada pelo teste de esfericidade de Bartlett (1954). Este teste estima os coeficientes das pontuações fatoriais. As pontuações resultantes possuem média igual a 0,00. Esse valor indica que as correlações entre as variáveis são significativas. Prova a hipótese nula: a matriz das correlações se ajusta entre as variáveis. Logo o determinante da matriz é um. Quando se aceita a hipótese nula $(\mathrm{p}>0,05)$, significa que as variáveis não estão intercorrelacionadas e, portanto, não existe sentido desenvolver uma análise fatorial.

O resultado do teste de Bartlett (1954) obtido nesse estudo foi considerado significativo $(\mathrm{p}<0,0001)$. Esse valor indicou que existe correlação entre as variáveis e estas são significativas. Expressa que as variáveis dependentes quantitativas presentes no Questionário que Diagnostica Práticas de Gestão Ambiental nos IF estão intercorrelacionadas e, portanto, existe sentido desenvolver uma análise fatorial. $\mathrm{O}$ valor significativo expressa que os 82 Campi analisados representam uma amostra aceitável.

O índice para o alfa de Cronbach do Questionário que analisa Práticas de Gestão Ambiental nos IF está especificado na Tabela 4.

Tabela 4 - Índice de confiabilidade do questionário que analisou práticas de gestão ambiental nos IF, Brasil, em 2010.

Table 4-Index of reliability of the questionnaire that examined environmental management practices in IF, Brazil, in 2010.

\begin{tabular}{cccc}
\hline $\begin{array}{c}\text { Campi } \\
\text { (população) }\end{array}$ & $\mathrm{n}$ (amostra) & $\alpha$ de Cronbach & No de items \\
\hline 230 & 82 & 0,5294 & 38 \\
\hline
\end{tabular}

Fonte: adaptado de Nogueiro (2008), usando o software R (R DEVELOPMENT CORE TEAM, 2007).

Foi avaliada a confiabilidade do Questionário que Diagnostica Práticas de Gestão Ambiental nos IF. A confiabilidade corresponde ao grau de coerência com o qual se mede a qualidade. Assim, analisou-se a consistência interna do questionário através do valor total do alfa $(\alpha)$ de Cronbach. Apresentado por Cronbach (1951), o coeficiente alfa de Cronbach é uma das estimativas da confiabilidade de um questionário que se aplica em uma pesquisa. Avalia a confiabilidade da pesquisa ou da análise dos itens na medição de um mesmo construto. O coeficiente varia de zero a um. Segundo Davis (1964), valores a partir de 0,50 são considerados aceitáveis.

Cerne, Lavras, v. 19, n. 2, p. 177-184, abr./jun. 2013 
O resultado do alfa de Cronbach obtido para a amostra de 82 Campi está dentro do mínimo recomendado por Davis (1964). Conforme esse autor, os critérios de recomendação de fiabilidade estimada pelo alfa de Cronbach devem ser superiores a 0,50. Já, Queiroga et al. (2006), estudando funções psicossociais sobre o comportamento do consumidor, encontraram alfa de Cronbach igual a 0,51.

Com base na avaliação do grau de implantação de determinadas vertentes e práticas de gestão ambiental foi traçado o IADAIFE de todos os Campi. O valor dos índices do IADAIFE variou de muito fraco a fraco. O menor valor obtido foi 0,17 (muito fraco: $0-0,20$ ) e o maior valor obtido foi 0,35 (fraco: 0,21-0,40), conforme Tabela 5 .

Tabela 5 - IADAIFE obtido para os 82 Campi dos IF analisados, Brasil, em 2010.

Table 5-IADAIFE obtained for 82 IF analyzed Campus, Brazil, in 2010 .

\begin{tabular}{lc}
\hline Campus & IADAIFE \\
\hline Colorado do Oeste RO & 0,17 \\
Iguatu CE & 0,17 \\
Araguatins TO & 0,18 \\
Nova Venécia ES & 0,20 \\
São Luiz Central MA & 0,21 \\
Rio do Sul SC & 0,21 \\
Catu BA & 0,21 \\
BJ Itabapoana RJ & 0,21 \\
Palmas TO & 0,21 \\
IFG Morrinhos 2 & 0,21 \\
Ipojuca PE & 0,22 \\
Rio Pomba MG & 0,22 \\
Senhor do Bonfim BA & 0,22 \\
São João Evangel MG & 0,22 \\
Barbacena MG & 0,22 \\
São Luiz MA & 0,22 \\
Alegrete 2 RS & 0,22 \\
João Pessoa PB & 0,23 \\
Caraguatatuba SP & 0,23 \\
Uberaba MG & 0,23 \\
Inhumas GO & 0,23 \\
\hline & Continua... \\
& \\
\hline &
\end{tabular}

To be continued...
Tabela 5 - Continuação...

Table 5 - Continued...

\begin{tabular}{|c|c|}
\hline Campus & IADAIFE \\
\hline Camaçari BA & 0,23 \\
\hline Videira SC & 0,23 \\
\hline Santa Inês BA & 0,23 \\
\hline Florianóp. Cons. SC & 0,23 \\
\hline Morrinhos GO & 0,23 \\
\hline Pelotas RS & 0,24 \\
\hline Bambuí MG & 0,24 \\
\hline Ouro Preto MG & 0,24 \\
\hline SJ Del Rey MG & 0,24 \\
\hline São Vicente MT & 0,24 \\
\hline Parnaíba PI & 0,24 \\
\hline Marabá PA & 0,24 \\
\hline Salvador BA & 0,24 \\
\hline Londrina PR & 0,24 \\
\hline Ceres GO & 0,24 \\
\hline Fortaleza CE & 0,26 \\
\hline Salinas MG & 0,26 \\
\hline São Gonçalo RJ & 0,26 \\
\hline Paraíso Tocantins TO & 0,26 \\
\hline Boa Vista RR & 0,26 \\
\hline Volta Redonda RJ & 0,26 \\
\hline Vitória ES & 0,26 \\
\hline São Roque SP & 0,26 \\
\hline Paracatu MG & 0,26 \\
\hline Uruçuca BA & 0,26 \\
\hline Santa Rosa RS & 0,26 \\
\hline Juazeiro Norte CE & 0,26 \\
\hline Nova Andradina MS & 0,26 \\
\hline Jaraguá do Sul SC & 0,27 \\
\hline Camboriú SC & 0,27 \\
\hline Guarulhos SP & 0,27 \\
\hline Congonhas MG & 0,27 \\
\hline Rio Grande RS & 0,27 \\
\hline Colatina ES & 0,27 \\
\hline Petrolina PE & 0,27 \\
\hline
\end{tabular}

Cerne, Lavras, v. 19, n. 2, p. 177-184, abr./jun. 2013 
Tabela 5 - Continuação...

Table 5 - Continued...

\begin{tabular}{|c|c|}
\hline Campus & IADAIFE \\
\hline Cáceres A MT & 0,27 \\
\hline Rio Verde GO & 0,27 \\
\hline Eunápolis BA & 0,28 \\
\hline Porto Alegre RS & 0,28 \\
\hline Concórdia SC & 0,28 \\
\hline Sapucaia do Sul RS & 0,28 \\
\hline Itumbiara GO & 0,28 \\
\hline Florianópolis SC & 0,28 \\
\hline Planaltina DF & 0,28 \\
\hline Bragança Paulista SP & 0,28 \\
\hline Alegrete RS & 0,28 \\
\hline Sombrio SC & 0,28 \\
\hline Uruaçu GO & 0,29 \\
\hline Picos PI & 0,29 \\
\hline Recife PE & 0,29 \\
\hline Curitiba PR & 0,29 \\
\hline Urutaí GO & 0,29 \\
\hline Formiga MG & 0,29 \\
\hline Araranguá SC & 0,30 \\
\hline Sobral CE & 0,30 \\
\hline Cáceres B MT & 0,30 \\
\hline Caicó RN & 0,32 \\
\hline Cuiabá MT & 0,32 \\
\hline Goiânia GO & 0,32 \\
\hline Cachoeiro Itapemi ES & 0,33 \\
\hline BV Cuiabá 2 MT & 0,35 \\
\hline
\end{tabular}

Fonte: dados da pesquisa usando o software R(RDEVELOPMENT CORE TEAM, 2007)

Esses resultados corroboraram os obtidos por Nogueiro (2008) que após analisar a Avaliação do Desempenho Ambiental dos Municípios (ADAM) para a amostra composta por 95 Municípios de Portugal, constatou que o desempenho ambiental dos municípios portugueses foi fraco. Analisando os valores do índice ADAM, o autor verificou que o desempenho ambiental dos municípios portugueses foi muito fraco $(51 \%)$ e fraco (21\%). A exceção foi para os municípios da região da capital Lisboa, que apresentaram desempenho ambiental médio.
Os resultados obtidos neste estudo também corroboraram os verificados por Ramos e Melo (2006) que após analisarem o desempenho ambiental das forças armadas de Portugal, verificaram que a integração de práticas ambientais no setor militar daquele país são atividades recentes. Os autores verificaram que o DAFA daquele país é fraco, com índice 0,33. Analisando individualmente cada setor das forças armadas, a força aérea portuguesa apresentou o melhor desempenho ambiental, com índice DAFA igual a 0,52, índice considerado médio. O exército apresentou DAFA igual a 0,30 , índice considerado fraco, e a marinha apresentou DAFA igual a 0,26 , índice considerado muito fraco.

Dos 82 Campi avaliados, 46,34\% disseram seguir os pressupostos da Lei oㅡ 9.795/1999; 48,78\% disseram não seguir; 4,88\% dos Campi disseram não saber (Tabela 6). Essa Lei estabelece os pressupostos sobre educação ambiental, institui a Política Nacional de Educação Ambiental e dá outras providências.

Tabela 6 - Índices percentuais dos Campi que seguiram quatro normas ambientais, que não seguiram e que não sabiam sobre quatro normas ambientais, Brasil, em 2010.

Table 6 - Percentage rates of campuses that followed, that not followed and that did not know about four environmental standards, Brazil, in 2010.

\begin{tabular}{lccc}
\hline Norma Ambiental & Sim (\%) & Não (\%) & Não sei (\%) \\
\hline -Resolução Conama & 37,80 & 58,50 & 3,70 \\
no 237/1997 & & & 4,89 \\
-Lei no 6.938/1981 & 37,80 & 57,31 & 7,32 \\
-Lei no 9.605/1998 & 42,68 & 50,00 & 4,88 \\
\hline -Lei no 9.795/1999 & 46,34 & 48,78 & \\
\hline
\end{tabular}

Fonte: dados da pesquisa utilizando o software R.2.10.1 (R DEVELOPMENT CORE TEAM, 2007).

\section{CONCLUSÕES}

O desempenho ambiental dos Institutos Federais de Educação, Ciência e Tecnologia avaliados no estudo pelo método IADAIFE apontou uma situação de baixa preocupação com o meio ambiente, ao afirmar que quatro Campi tiveram desempenho ambiental muito fraco e 78 Campi fracos, em escala que o desempenho ambiental variava entre muito fraco, fraco, médio, bom e muito bom.

Existem Campi que não dispõem de estrutura responsável exclusivamente pelas questões ambientais, cabendo a outros setores essa função. É possível concluir que a questão ambiental ainda é deixada de lado para parte dos IF brasileiros.

Cerne, Lavras, v. 19, n. 2, p. 177-184, abr./jun. 2013 
Houve dificuldades para os Campi seguirem os desígnios legais de quatro normas ambientais: Resolução Conama no 237/1997, Lei no 6.938/1981, Lei no 9.605/1998 e Lei no 9.795/1999, inclusive por desconhecimento da norma ambiental. Esta situação pode acarretar na formação de futuros profissionais sem uma formação ambiental mínima, o que pode acarretar em mais uma geração de profissionais céticos à proteção ambiental.

Devem ser adotadas novas práticas e políticas públicas para que se inverta a tendência que demonstrou o desempenho ambiental dos IF, principalmente por serem instituições promovedoras e replicadoras de conhecimento.

\section{REFERÊNCIAS}

ASSOCIAÇÃO BRASILEIRA DE NORMAS TÉCNICAS. NBR ISO 14031: gestão ambiental: avaliação do ciclo de vida ambiental. Rio de Janeiro, 1999.

BARATA, M. L. L.; GOMEZ, C. M.; CLIGERMAN, D. C. A gestão ambiental no setor público: uma questão de relevância social e econômica. Revista Ciência \& Saúde Coletiva, Brasília, v. 12, n. 1, p. 165-170, jan. 2007.

BARTLETT, M. S. A norte of the multiplying factors for various chi square approximations. Journal of the Royal Statistical Society: Series B, Statistical Methodology, Oxford, v. 16, n. 1, p. 296-298, 1954.

BONNET, J. F. Analysis of electricity and water end-uses in university campuses: case-study of the University of Bordeaux in the framework of the Ecocampus European Collaboration. Journal of Cleaner Production, Amsterdam, v. 10, n. 1, p. 13-24, Feb. 2002.

CRONBACH, L. J. Coefficient alpha and the internal structure of tests. Revista Psychometrika, Stockholm, v. 16, p. 297-337, 1951. Disponível em: <http://www.springerlink.com/content/ n435u12541475367/>. Acesso em: 4 dez. 2011.

DAVIS, F. B. Educational measurements and their interpretation. Belmont: Wadsworth, 1964.

DRUZZIAN, E. T. V.; SANTOS, R. C. Sistema de gerenciamento ambiental: buscando uma resposta para os resíduos de laboratórios das instituições de ensino médio e profissionalizante. Revista Liberato, Porto Alegre, v. 7, n. 7, p. 40-44, jan. 2006.

FLORIANO, E. P. Políticas de gestão ambiental. 3. ed. Florianópolis: UFSM, 2007.

Cerne, Lavras, v. 19, n. 2, p. 177-184, abr./jun. 2013
GOMES, W. C. Avaliação institucional: gestão do Programa de Avaliação Institucional das Universidades Brasileiras-PAIUB. 2006. 222 p. Dissertação (Mestrado em Ciências Sociais) Universidade Federal do Maranhão, São Luiz, 2006.

GROBER, U. Von kursachsen Nach Rio: ein lebensbild über den erfinder der nachhaltigkeit Hannß Carl Edler von Carlowitz und die Wegbeschreibung eines Konzeptes: aus der Silberstadt Freiberg. Disponível em: <http://www.forschungsheim.de/ fachstelle/arb_carl .htm>. Acesso em: 5 dez. 2010.

NOGUEIRO, L. A. S. Práticas de gestão ambiental na administração pública local. 2008. 136 p. Dissertação (Mestrado em Ciências e Engenharia do Ambiente) - Universidade Nova de Lisboa, Lisboa, 2008.

QUEIROGA, F.; GOUVEIA, V. V.; COUTINHO, M. P. L. Intenção de comportamento socialmente responsável do consumidor: sua relação com os valores humanos básicos. PsicoUSF, Itatiba, v. 11, n. 2, p. 239-248, dez. 2006.

R DEVELOPMENT CORE TEAM. R: a language and environment for statistical computing. Vienna: R Foundation for Statistical Computing, 2007. Disponível em: $<$ http://www.rproject.org >. Acesso em: 10 mar. 2010.

RAMOS, T. B. Avaliação do desempenho ambiental no sector público: estudo do sector da defesa. 2004. 164 p. Tese (Doutorado em Ciências e Tecnologia) - Universidade Nova de Lisboa, Lisboa, 2004.

RAMOS, T. B.; ALVES, I.; SUBTIL, R.; MELO, J. J. The state of environmental performance evaluation in the public sector: the case of the Portuguese defense sector. Journal of Cleaner Production, New York, v. 17, p. 36-52, 2009. Disponível em: <http://www. elsevier.com/locate/jclepro>. Acesso em: 17 mar. 2012.

RAMOS, T. B.; MELO, J. J. Developing and implementing an environmental performance index for the portuguese military. Business Strategy and the Environment, Chicago, v. 15, p. 71-86, 2006. Disponível em: <http://www.interscience.wiley. com>. Acesso em: 17 mar. 2012.

THE SWANSEA declaration. Swansea, 1993. Disponível em: $<$ http://www.iisd.org.htm>. Acesso em: 18 nov. 2009.

TAUCHEN, J.; BRANDLI, L. L. A gestão ambiental em instituições de ensino superior: modelo para implantação em campus universitário. Revista Gestão e Produção, São Carlos, v. 13, n. 3, p. 503-515, set./dez. 2006. 